\title{
Prediction for Contrast Volume in Transcatheter Aortic Valve Replacement - Important but Modifiable?
}

\author{
Jolanta M. Siller-Matula a, b Mamas A. Mamas ${ }^{c, d}$ \\ a Division of Cardiology, Department of Internal Medicine II, Medical University of Vienna, Vienna, Austria; \\ ${ }^{b}$ Department of Experimental and Clinical Pharmacology, Centre for Preclinical Research and Technology (CEPT), \\ Medical University of Warsaw, Warsaw, Poland; 'Keele Cardiovascular Research Group, Keele University, Keele, UK; \\ ${ }^{d}$ Royal Stoke University Hospital, Stoke-on-Trent, UK
}

Procedural safety in patients undergoing transcatheter aortic valve replacement (TAVR) has improved over the recent years, particularly with the developing evidence basis of the efficacy of TAVR in lower-risk patients [1]. Nevertheless, a significant proportion of patients undergoing TAVR are elderly and multimorbid. Up to $30-50 \%$ of this population have documented chronic kidney disease (CKD), which together with other clinical comorbidities and pharmacological treatments of severe aortic stenosis (e.g., diuretic administration) increases the risk of developing acute kidney injury (AKI), particularly following procedures involving the use of intravascular contrast agents. Contrast-induced AKI (CIAKI) remains one of the strongest predictors of short- and long-term mortality after TAVR [2]. Therefore, TAVR, especially in patients with CKD, represents a challenge due to the high risk of CIAKI.

In this issue of Cardiology, Goldsweig et al. [3] present an analysis focusing on the characterization of predictors of overall contrast volume administered during the procedure in 295 TAVR patients at a single US center from 2013 to 2018. The authors identified independent predictors of overall contrast volume use in their cohort, in which the median contrast volume was reported as 150
$\mathrm{mL}$. The predictors of contrast volume use during TAVR identified in the study by Goldsweig et al. [3] warrant discussion to place these results into perspective. Amongst the strongest predictors of contrast volume was found to be procedure year 2016 versus 2018 that had a beta weight of 44.2 (95\% confidence interval 16.0-72.4). This would suggest a significant learning curve with TAVR: over time, operators tended to use less contrast volume, but it may also be a surrogate for less complex procedures undertaken in a lower risk population over time as the indications for TAVR expand $[1,4,5]$.

Moreover, the study by Goldsweig et al. [3] suggests that TAVR device type did not predict the contrast volume used. This is an interesting finding, which would implicate that the device type should not correlate with CIAKI. Indeed, in the low-risk TAVR population, TAVR was associated with a $73 \%$ relative risk reduction of developing AKI as compared to surgical aortic valve replacement, but there was no difference in the magnitude of the effect between the different TAVR valve types (self-expanding Medtronic valve vs. balloon-expandable Edwards valve) [1].

Nevertheless, the study by Goldsweig et al. [3] has the limitation of a small sample size and a single-center de- karger@karger.com www.karger.com/crd

(c) 2020 S. Karger AG, Basel

Karger"
Jolanta M. Siller-Matula

Department of Internal Medicine II, Division of Cardiology Medical University of Vienna, Währinger Gürtel 18-20 AT-1090 Vienna (Austria)

Jolanta.siller-matula@meduniwien.ac.at 
sign. Furthermore, it is unclear from this study whether coronary angiography/percutaneous coronary intervention was undertaken at the same time as the TAVR procedure and whether this contributed to contrast load, or whether these procedures were performed a priori. This would be important determinants of contrast volume. Indeed, in the advent of coronary computed tomography (CT) as a modality to define coronary and valvular anatomy, and limited data around the usefulness of coronary revascularization prior to TAVR [6], contrast volume in TAVR procedures can be further minimized by avoiding potentially unnecessary procedures. Finally, another important unanswered question relates to the clinical outcomes of patients in the study by Goldsweig et al. [3], and what proportion of them actually sustained CIAKI, as contrast volume is only one of many determinants of this important complication. Indeed, a previous metanalysis of 4 studies recruiting 891 patients showed no significant association between contrast media volume and risk of AKI after TAVR [7].

Identification of patients at highest risk of contrastinduced nephropathy, and ensuring adequate pre-hydration, and minimization of contrast use in such patients remain important strategies in preventing CIAKI. The question remains which further actions can be undertaken to minimize contrast volume use in this at-risk population? Unfortunately, it has been suggested that all formulations of contrast media are cytotoxic. Free iodine from the contrast has a direct cytotoxic effect on the endothelial cells and on the tubules of the nephron $[8,9]$. During this process, reactive oxygen species are produced, which consume $\mathrm{NO}$ and prevent its protective effect as a vasodilator $[10,11]$. As a consequence, vasoconstriction decreases glomerular filtration rate, increases blood viscosity, and causes medullary hypoperfusion [9]. It has been shown that the total antioxidant capacity decreases immediately after TAVR and returns to baseline on average 2 days after the procedure [12]. However, it is unclear to which extent the overall contrast volume impacts the total antioxidant capacity and markers of redox state after TAVR.

Previously, a strategy of furosemide-induced diuresis with matched isotonic intravenous hydration in the PROTECT-TAVI trial (PROphylactic effecT of furosEmideinduCed Diuresis with Matched Isotonic Intravenous hydraTion in Transcatheter Aortic Valve Implantation) using the RenalGuard system has been shown to decrease the incidence of CIAKI in patients undergoing TAVR, although no differences in mortality, cerebrovascular events, bleeding, and hospitalization for heart failure were noted in both groups at 30 days [13]. More recently, a novel strategy of "zero-contrast" TAVR in patients with advanced CKD preceded by accurate CT scan analysis and procedural planning was proposed and shown to be safe and feasible [14]. Twelve patients suffering from at least stage $4 \mathrm{CKD}$ underwent both screening with preprocedural CT scan and bioprosthetic valve implantation without contrast medium. All TAVR procedures were performed using the CoreValve Evolut transcatheter aortic valve. The annulus and the optimal implantation projection were identified on the non-contrast medium CT scans with the aid of calcification as a reference. The implant projection was confirmed immediately before valve implantation by placing two pigtail catheters alternately inside each sinus of Valsalva. The study showed that no patient had more than a mild paravalvular leak, and none of the patients developed AKI.

Clearly, not all patients undergoing TAVR are at high risk of developing CIAKI. Whilst risk scores have been published to predict CIAKI in patients undergoing percutaneous coronary intervention [15] who have been validated externally, there are limited robust prognostic models for the prediction of CIAKI in patients undergoing TAVR, particularly given the rapidly evolving technologies involved and the types/risk profile of patients who undergo these procedures. Future work should focus on the development of such prognostic models that will aid in the identification of patients at high risk for sustaining AKI, where contrast volume is likely to be one of many factors that will be identified, so that steps can be taken to mitigate risk in high-risk patients identified using these tools.

\section{Disclosure Statement}

J.M.S.-M received speaker honoraria from Chiesi, Bayer, Daiichi-Sankyo, and BMS. M.A.M has no conflicts to declare for the current publication.

\section{References}

Hofer F, Hengstenberg C, Goliasch G, Grygier M, Mascherbauer J, Siller-Matula JM. Transcatheter versus surgical aortic valve replacement in low-risk patients: a meta-analysis of randomized trials. Clin Res Cardiol. 2020 Jun;109(6):761-75.

2 Bagur R, Webb JG, Nietlispach F, Dumont E, De Larochellière R, Doyle D, et al. Acute kidney injury following transcatheter aortic valve implantation: predictive factors, prognostic value, and comparison with surgical aortic valve replacement. Eur Heart J. 2010 Apr; 31(7):865-74. 
3 Goldsweig AM, Lyden E, Aronow HD, Kolte D, Pavlides G, Barton D, et al. Predictors of Contrast Volume in Transcatheter Aortic Valve Replacement. Cardiology. DOI: $10.1159 / 000507506$.

4 Kim WK, Möllmann H, Liebetrau C, Renker M, Rolf A, Simon P, et al. The Acurate neo transcatheter heart valve: a comprehensive analysis of predictors of procedural outcome. JACC Cardiovasc Interv. 2018 Sep;11(17): 1721-9.

5 Hengstenberg C, Siller-Matula JM. Coming Closer to Personalized Medicine in Transcatheter Aortic Valve Replacement. JACC Cardiovasc Interv. 2018 Sep;11(17):1730-2.

6 Kotronias RA, Kwok CS, George S, Capodanno D, Ludman PF, Townend JN, et al. Transcatheter Aortic Valve Implantation With or Without Percutaneous Coronary Artery Revascularization Strategy: A Systematic Review and Meta-Analysis. J Am Heart Assoc. 2017 Jun;6(6):e005960.

7 Thongprayoon C, Cheungpasitporn W, Podboy AJ, Gillaspie EA, Greason KL, Kashani KB. The effects of contrast media volume on acute kidney injury after transcatheter aortic valve replacement: a systematic review and meta-analysis. J Evid Based Med. 2016 Nov; 9(4):188-93.

8 Mamoulakis C, Fragkiadoulaki I, Karkala P, Georgiadis G, Zisis IE, Stivaktakis P, et al. Contrast-induced nephropathy in an animal model: evaluation of novel biomarkers in blood and tissue samples. Toxicol Rep. 2019 May;6:395-400.

9 Morcos R, Kucharik M, Bansal P, Al Taii H, Manam R, Casale J, et al. Contrast-Induced Acute Kidney Injury: Review and Practical Update. Clin Med Insights Cardiol. 2019 Nov; 13:1179546819878680.

10 Mamoulakis C, Tsarouhas K, Fragkiadoulaki I, Heretis I, Wilks MF, Spandidos DA, et al. Contrast-induced nephropathy: basic concepts, pathophysiological implications and prevention strategies. Pharmacol Ther. 2017 Dec;180:99-112.

11 Komosa A, Rzymski P, Perek B, RopackaLesiak M, Lesiak M, Siller-Matula JM, et al. Platelets redox balance assessment: current evidence and methodological considerations. Vascul Pharmacol. 2017 Aug;93-95:6-13.

12 Komosa A, Perek B, Rzymski P, Lesiak M, Siller-Matula JM, Grygier M, et al. Transcatheter Aortic Valve Replacement Is Associated with Less Oxidative Stress and Faster Recovery of Antioxidant Capacity than Surgical Aortic Valve Replacement. J Clin Med. 2019 Sep;8(9):E1364.

13 Barbanti M, Gulino S, Capranzano P, Immè S, Sgroi C, Tamburino C, et al. Acute Kidney Injury With the RenalGuard System in Patients Undergoing Transcatheter Aortic Valve Replacement: the PROTECT-TAVI Trial (PROphylactic effecT of furosEmide-induCed diuresis with matched isotonic intravenous hydraTion in Transcatheter Aortic Valve Implantation). JACC Cardiovasc Interv. 2015 Oct;8(12):1595-604.

14 Maffeo D, Bettari L, Latib A, Maiandi C, Villa E, Messina A, et al. Transfemoral transcatheter aortic valve replacement without contrast medium using the Medtronic CoreValve system: a single center experience. J Cardiovasc Surg (Torino). 2020 Apr. Online ahead of print.

15 Mehran R, Aymong ED, Nikolsky E, Lasic Z, Iakovou I, Fahy M, et al. A simple risk score for prediction of contrast-induced nephropathy after percutaneous coronary intervention: development and initial validation. J Am Coll Cardiol. 2004 Oct;44(7):1393-9. 